\title{
Sarcopenia Is Independently Associated with the Degree of Liver Fibrosis in Patients with Type 2 Diabetes Mellitus
}

\author{
Min Je Sung ${ }^{1}$, Tae Seop Lim ${ }^{1,2}$, Mi Young Jeon ${ }^{1,2}$, Hye Won Lee ${ }^{1,2}$, Beom Kyung Kim ${ }^{1,2,3}$, Do Young Kim ${ }^{1,2,3}$, Sang Hoon \\ $\mathrm{Ahn}^{1,2,3}$, Kwang-Hyub Han ${ }^{1,2,3}$, Jun Yong Park ${ }^{1,2,3}$, and Seung Up Kim ${ }^{1,2,3}$ \\ ${ }^{1}$ Department of Internal Medicine, Yonsei University College of Medicine, ${ }^{2}$ Yonsei Liver Center, Severance Hospital, and ${ }^{3}$ Institute of \\ Gastroenterology, Yonsei University College of Medicine, Seoul, Korea
}

Background/Aims: Sarcopenia is associated with liver fibrosis in patients with nonalcoholic fatty liver disease and chronic hepatitis B. We investigated the association between sarcopenia and hepatic fibrotic burden in patients with type 2 diabetes mellitus (T2DM). Methods: Patients with T2DM who had received a comprehensive medical health checkup were recruited. Muscle mass was assessed using computed tomography. Fibrotic burden was assessed using the fibrosis-4 index (FIB-4). The study population was divided by quartile stratification of the lumbar skeletal muscle index (LSMI). Results: Among 309 patients with T2DM, 75 (24.3\%) had sarcopenia. These patients were significantly older and had higher FIB-4, whereas they had significantly lower body mass index (BMI) and LSMI than patients without sarcopenia (all $p<0.05$ ). The LSMI showed a significant negative correlation with the FIB-4 when analyzed in terms of quartile stratification ( $p=0.003)$. Multivariate analysis showed that female sex and higher BMI were independently associated with a reduced risk of sarcopenia (odds ratio [OR], 0.388; 95\% confidence interval [Cl], 0.199 to 0.755 and OR, 0.704; $95 \% \mathrm{Cl}, 0.618$ to 0.801 ; all $\mathrm{p}<0.05)$, whereas a higher FIB4 was independently associated with an increased risk of sarcopenia (OR, 1.817; 95\% $\mathrm{Cl}, 1.180$ to 2.797 ; $p=0.007$ ). Among patients with a BMl $<25 \mathrm{~kg} / \mathrm{m}^{2}(\mathrm{n}=165)$, those with sarcopenia ( $n=54,32.7 \%)$ had a significantly higher FIB-4 than those without $(n=111,67.3 \% ; 1.66$ vs $1.38, p=0.004)$. Conclusions: Sarcopenia is independently associated with fibrotic burden in patients with T2DM. Further studies should investigate whether the improvement of sarcopenia can ameliorate liver fibrosis in patients with T2DM. (Gut Liver 2020;14:626-635)
Key Words: Sarcopenia; Liver fibrosis; Muscular atrophy; Diabetes mellitus; Fibrosis-4 index

\section{INTRODUCTION}

Sarcopenia is characterized by progressive and generalized loss of skeletal muscle mass and strength, with a risk of adverse outcomes, such as physical disability, poor quality of life, and death, according to the 2010 European Working Group on Sarcopenia in Older People. ${ }^{1}$ In fact, sarcopenia has emerged as one of the most common problems in the elderly population, and it is one of the most significant public health concerns. ${ }^{2}$ Thus, the European Working Group on Sarcopenia in Older People emphasized that clinicians must measure and evaluate muscle mass, muscle strength, and physical performance to diagnose sarcopenia. $^{1}$

Low skeletal muscle mass can be evaluated using various imaging techniques, such as dual-energy X-ray absorptiometry (DXA), computed tomography (CT), magnetic resonance imaging, and bioimpedance analysis (BIA). Among these methods, CT images allow accurate quantitative and qualitative measurement of body composition, including adipose tissue. ${ }^{1}$ Additionally, skeletal muscle mass measured by CT, rather than BIA, is significantly correlated with muscle strength and physical performance. $^{3}$

Sarcopenia is often combined with various comorbidities, ${ }^{4}$ including several cardiometabolic disorders such as diabetes mellitus, metabolic syndrome, cardiovascular disease, and nonalcoholic fatty liver disease (NAFLD), ${ }^{5-8}$ which has become a major liver disease world-wide. ${ }^{9}$ Several recent studies have reported that sarcopenia is significantly associated with an increased risk of significant liver fibrosis in subjects with NAFLD. ${ }^{10-13}$ In addi-

Correspondence to: Jun Yong Park ${ }^{\mathrm{a}}$ and Seung Up Kim ${ }^{\mathrm{b}}$

Department of Internal Medicine, Yonsei University College of Medicine, 50 Yonsei-ro, Seodaemun-gu, Seoul 03722, Korea

aTel: +82-2-2228-1944, Fax: +82-2-393-6884, E-mail: DRPJY@yuhs.ac

'Tel: +82-2-2228-1988, Fax: +82-2-393-6884, E-mail: ksukorea@yuhs.ac

Received on April 9, 2019. Revised on August 26, 2019. Accepted on September 19, 2019. Published online March 6, 2020. pISSN 1976-2283 eISSN 2005-1212 https://doi.org/10.5009/gnl19126

(a) This is an Open Access article distributed under the terms of the Creative Commons Attribution Non-Commercial License (http://creativecommons.org/licenses/by-nc/4.0) which permits unrestricted non-commercial use, distribution, and reproduction in any medium, provided the original work is properly cited. 
tion, in patients with chronic hepatitis B who have metabolically unhealthy features, sarcopenia is significantly associated with an increased risk of significant liver fibrosis similar to NAFLD. ${ }^{14}$ However, few studies have addressed the relationship between sarcopenia and liver fibrosis in patients with type 2 diabetes mellitus (T2DM).

Thus, the aim of the present study was to compare clinical characteristics between patients with and without sarcopenia, as assessed using CT, as well as to investigate whether sarcopenia is associated with the degree of liver fibrosis in patients with T2DM.

\section{MATERIALS AND METHODS}

\section{Patients}

Between June 2010 and April 2018, we recruited 1,191 subjects who had visited Severance Executive Healthcare Clinic in Severance Hospital, a university-affiliated tertiary care hospital, for a comprehensive medical health check-up over a 2-day hospitalization period (Fig. 1). Of these, 868 were excluded because they had no available CT scan or did not have T2DM. ${ }^{15}$ Fourteen more were then excluded based on our exclusion criteria. Ultimately, 309 patients with T2DM (215 men and 94 women) were selected for the statistical analysis.

The protocol of the present study adhered to the tenets of the Declaration of Helsinki and was approved by the Institutional Review Board of Severance Hospital (IRB number: 4-20180856). The requirement for informed consent was waived because the study was retrospective.

\section{Measurements of skeletal muscle area}

Skeletal muscle area was measured at the level of the L3 vertebra, with the patients in the supine position, using either a dual-source, 128-slice CT scanner (Somatom Definition Flash; Siemens Healthcare, Forchheim, Germany), a 64-slice CT scanner (Somatom Sensation 64; Siemens Healthcare), a 128 slice Discovery 710 PET-CT scanner (General Electric Medical Systems, Milwaukee, WI, USA), a 40 slice Biograph TruePoint 40 PET-CT scanner (Siemens Medical Solutions, Hoffman Estates, IL, USA), or a 16 slice Discovery 600 PET-CT scanner (General Electric Medical Systems). The muscle area was identified as having attenuation values between -29 and 150 Hounsfield units, and the total lumbar skeletal muscle area (psoas, erector spinae, quadratus lumborum, transversus abdominus, external and internal obliques, and rectus abdominus), measured in $\mathrm{cm}^{2}$, was defined as the region with density ranging from -29 to -150 Hounsfield units, as measured using Aquarius Intuition Viewer software, version 4.4.12 (Terarecon, San Mateo, CA, USA). Boundaries were corrected manually as necessary. To determine the lumbar skeletal muscle index (LSMI; $\mathrm{cm}^{2} / \mathrm{m}^{2}$ ), the skeletal muscle area at the L3 vertebra was normalized to the square of the patient's height $\left(\mathrm{m}^{2}\right)^{16,17}$

\section{Definition of T2DM and sarcopenia}

Diabetes mellitus was identified when patients used insulin or oral hypoglycemic agents or had a fasting plasma glucose level $\geq 126 \mathrm{mg} / \mathrm{dL} .{ }^{18}$ Sarcopenia was defined as the lowest LSMI quartile in our study participants, corresponding to cutoff points of $48.10 \mathrm{~cm}^{2} / \mathrm{m}^{2}$ in men and $36.04 \mathrm{~cm}^{2} / \mathrm{m}^{2}$ in women.

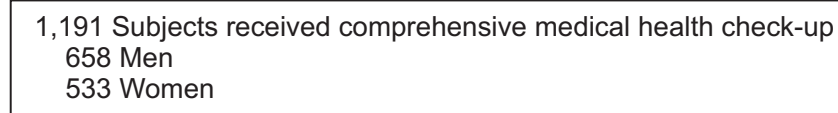

\begin{tabular}{|c|c|}
\hline & $\begin{array}{l}\text { Non available CT scan } \\
\text { No diabetes }\end{array}$ \\
\hline $\begin{array}{l}323 \text { Subjects with diabetes and m } \\
227 \text { Men } \\
96 \text { Women }\end{array}$ & \\
\hline
\end{tabular}

Fig. 1. Flow of study population selection. CT, computed tomography; HBV, hepatitis B virus; HCV, hepatitis C virus; HIV, human immunodeficiency virus. 


\section{Assessment of liver fibrosis and steatosis}

In all participants, the fibrotic burden in the liver was assessed using the fibrosis-4 (FIB-4) index and NAFLD fibrosis score (NFS), which are simple, noninvasive fibrosis scoring systems. ${ }^{19,20}$ The FIB-4 index was calculated using the following formula: ${ }^{19}$ (age [years]) $\times($ aspartate aminotransferase [AST] level [U/L])/(platelet count $\left.\left[10^{9} / \mathrm{L}\right]\right) \times\left(\right.$ alanine aminotransferase $[\text { ALT] level }[\mathrm{U} / \mathrm{L}])^{1 / 2}$. NFS was calculated using the following formula: $-1.675+(0.037 \times$ age [years])+(0.094×BMI $\left.\left[\mathrm{kg} / \mathrm{m}^{2}\right]\right)+(1.13 \times$ impaired glucose tolerance/ diabetes [yes $=1$, no $=0])+(0.99 \times$ AST/ALT ratio $)-(0.013 \times$ platelet count $\left.\left[\times 10^{9} / \mathrm{L}\right]\right)-(0.66 \times$ serum albumin $[\mathrm{g} / \mathrm{dL}]){ }^{20}$

Subgroup analysis was performed in patients with available transient elastography (TE) evaluation (FibroScan; EchoSens, Paris, France). The principles of measuring liver stiffness (LS) and controlled attenuation parameter using TE have been described previously. ${ }^{21,22}$ The LS results are expressed in terms of kilopascal, while the controlled attenuation parameter results are given in $\mathrm{dB} / \mathrm{m}$. To estimate variability, we calculated the ratio of the LS interquartile range (IQR) to its median. Only a valid LS of $\geq 10 \mathrm{kPa}$, a success rate of $\geq 60 \%$, and a ratio of IQR to median LS of $\leq 30 \%$ were deemed reliable. The controlled attenuation parameter was considered a valid value only when the LS of the same signal was reliable.

\section{Statistical analysis}

The characteristics of the study subjects were analyzed in terms of their sarcopenia status using the Student t-test for continuous variables and the chi-square test for categorical variables. The association between LSMI and fibrotic burden represented using FIB-4 index and NFS values was evaluated using simple linear regression analysis and the chi-square test after transformation of these variables into quartiles. Multivariable logistic regression analysis was applied to determine the independent association between sarcopenia and other variables. Continuous variables were expressed as the mean \pm standard deviation, while categorical variables were given in terms of number (\%). p-values $<0.05$ were considered statistically significant. Statistical analyses were performed using SPSS version 23.0 for Windows (IBM Corp., Armonk, NY, USA).

Table 1. Baseline Characteristics of the Study Population

\begin{tabular}{|c|c|c|c|c|}
\hline Variable & $\begin{array}{c}\text { All } \\
(\mathrm{n}=309)\end{array}$ & $\begin{array}{l}\text { Non-sarcopenic } \\
(\mathrm{n}=234,75.7 \%)\end{array}$ & $\begin{array}{c}\text { Sarcopenic } \\
(\mathrm{n}=75,24.3 \%)\end{array}$ & $\mathrm{p}$-value \\
\hline \multicolumn{5}{|l|}{ Demographic parameter } \\
\hline Age, yr & $62.7 \pm 10.5$ & $60.9 \pm 10.0$ & $68.2 \pm 10.2$ & $<0.001$ \\
\hline Female sex & $94(30.4)$ & $76(32.5)$ & $18(24.0)$ & 0.165 \\
\hline Body mass index, $\mathrm{kg} / \mathrm{m}^{2}$ & $25.3 \pm 2.9$ & $25.7 \pm 2.9$ & $23.8 \pm 2.3$ & $<0.001$ \\
\hline Hypertension & $222(71.8)$ & $169(72.2)$ & $53(70.7)$ & 0.794 \\
\hline \multicolumn{5}{|l|}{ Laboratory parameter } \\
\hline Fasting glucose, mg/dL & $122 \pm 37$ & $122 \pm 36$ & $122 \pm 43$ & 0.933 \\
\hline Aspartate aminotransferase, IU/L & $24.6 \pm 10.8$ & $24.9 \pm 11.3$ & $23.5 \pm 9.2$ & 0.326 \\
\hline Alanine aminotransferase, IU/L & $25.9 \pm 14.2$ & $27.0 \pm 15.2$ & $22.7 \pm 9.7$ & 0.005 \\
\hline Gamma glutamyl-transpeptidase, IU/L & $42 \pm 48$ & $45 \pm 51$ & $34 \pm 37$ & 0.086 \\
\hline Serum creatinine, $\mathrm{mg} / \mathrm{dL}$ & $0.9 \pm 0.6$ & $0.9 \pm 0.4$ & $1.0 \pm 0.8$ & 0.197 \\
\hline Platelet count, $10^{9} / \mathrm{L}$ & $228 \pm 58$ & $230 \pm 60$ & $222 \pm 48$ & 0.284 \\
\hline Prothrombin time, INR & $0.94 \pm 0.20$ & $0.93 \pm 0.07$ & $0.99 \pm 0.39$ & 0.028 \\
\hline Total cholesterol, mg/dL & $179 \pm 42$ & $181 \pm 42$ & $173 \pm 40$ & 0.192 \\
\hline Triglycerides, mg/dL & $142 \pm 86$ & $147 \pm 92$ & $126 \pm 63$ & 0.069 \\
\hline High-density lipoprotein cholesterol, mg/dL & $46 \pm 11$ & $46 \pm 12$ & $47 \pm 11$ & 0.774 \\
\hline Low-density lipoprotein cholesterol, mg/dL & $104 \pm 35$ & $105 \pm 35$ & $100 \pm 35$ & 0.386 \\
\hline $\mathrm{HbA} 1 \mathrm{c}, \%$ & $6.7 \pm 1.1$ & $6.7 \pm 1.1$ & $6.8 \pm 1.3$ & 0.234 \\
\hline \multicolumn{5}{|l|}{ Muscle mass parameter } \\
\hline L3 skeletal muscle area, $\mathrm{cm}^{2}$ & $139 \pm 34$ & $146 \pm 33$ & $117 \pm 25$ & $<0.001$ \\
\hline Lumbar skeletal muscle index, $\mathrm{cm}^{2} / \mathrm{m}^{2}$ & $50 \pm 10$ & $53 \pm 9$ & $41 \pm 7$ & $<0.001$ \\
\hline \multicolumn{5}{|l|}{ Fibrosis parameter } \\
\hline Fibrosis-4 index & $1.46 \pm 0.64$ & $1.41 \pm 0.63$ & $1.63 \pm 0.65$ & 0.009 \\
\hline Liver stiffness, $\mathrm{kPa}(\mathrm{n}=113)$ & $5.2 \pm 3.1$ & $4.9 \pm 2.7(n=93)$ & $6.4 \pm 1.0(\mathrm{n}=20)$ & 0.159 \\
\hline
\end{tabular}

Data are presented as mean \pm SD or number (\%).

INR, international normalized ratio; HbA1c, glycosylated hemoglobin. 


\section{RESULTS}

\section{Subject characteristics}

The baseline characteristics of the study population are summarized in Table 1 . The mean age of the study population was 62.7 years, while their mean body mass index (BMI) was 25.3 $\mathrm{kg} / \mathrm{m}^{2}$. Their mean fasting glucose was $122 \mathrm{mg} / \mathrm{dL}$, while their mean $\mathrm{HbA1c}$ (glycosylated hemoglobin) was 6.7\%. Their mean total cholesterol, triglyceride, high-density lipoprotein cholesterol, and low-density lipoprotein cholesterol were 179, 142, 46, and $104 \mathrm{mg} / \mathrm{dL}$, respectively. Their mean L3 skeletal muscle area was $139 \mathrm{~cm}^{2}$, while their mean LSMI was $50 \mathrm{~cm}^{2} / \mathrm{m}^{2}$. The mean FIB-4 index and NFS in the entire population were 1.46 and
-0.587, respectively, and the mean LS value was $5.2 \mathrm{kPa}$ in the subgroup whose LS value was available $(n=113)$.

\section{Comparison between subjects with sarcopenia and those without}

Of the study participants, 75 (24.3\%) had sarcopenia. Subjects with sarcopenia were significantly older than those without (mean, 68.2 years vs 60.9 years) (Table 1), and they had significantly lower BMI (mean, $23.8 \mathrm{~kg} / \mathrm{m}^{2}$ vs $25.7 \mathrm{~kg} / \mathrm{m}^{2}$; all p<0.05). In addition, subjects with sarcopenia had significantly lower ALT levels (mean, 22.7 IU/L vs 27.0 IU/L), as well as significantly prolonged prothrombin time (mean international normalized ratio, 0.99 vs 0.93 ) than those without sarcopenia (all $p<0.05$ ),
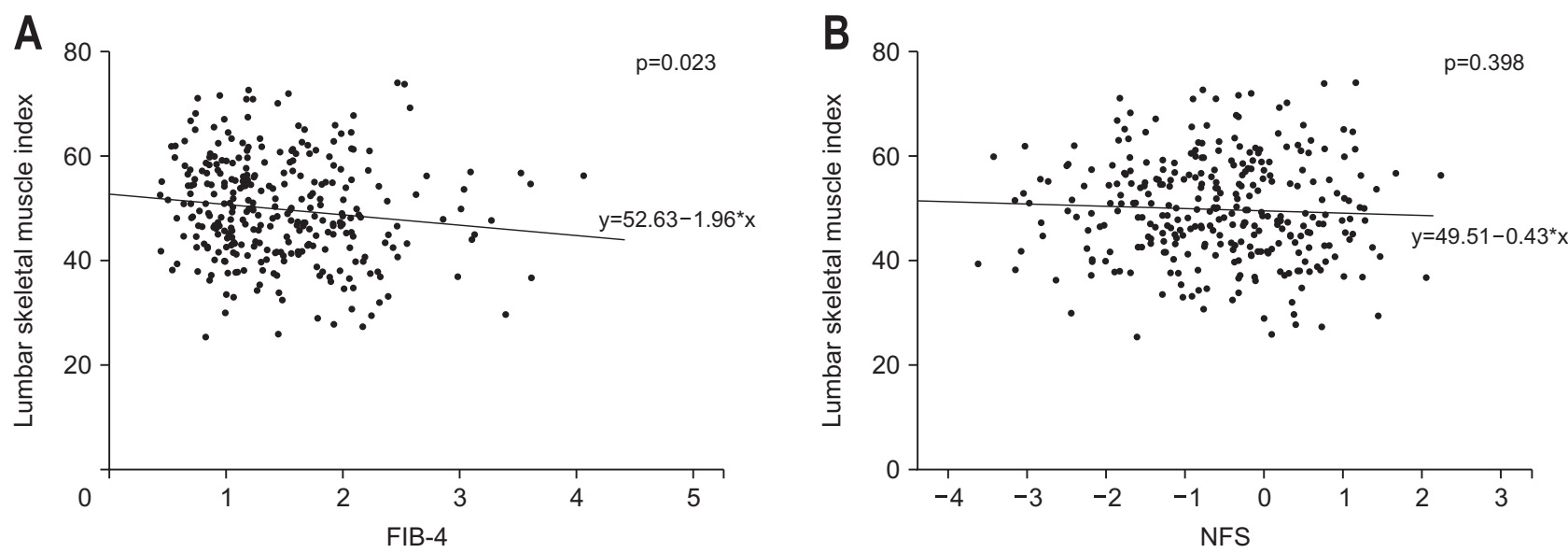

Fig. 2. Association between muscle mass and fibrotic burden using the fibrosis-4 (FIB-4) index (A) and NAFLD fibrosis score (NFS) (B). The muscle mass showed a strong negative association with fibrotic burden using the FIB-4 index ( $\mathrm{p}=0.023)$. NAFLD, nonalcoholic fatty liver disease.
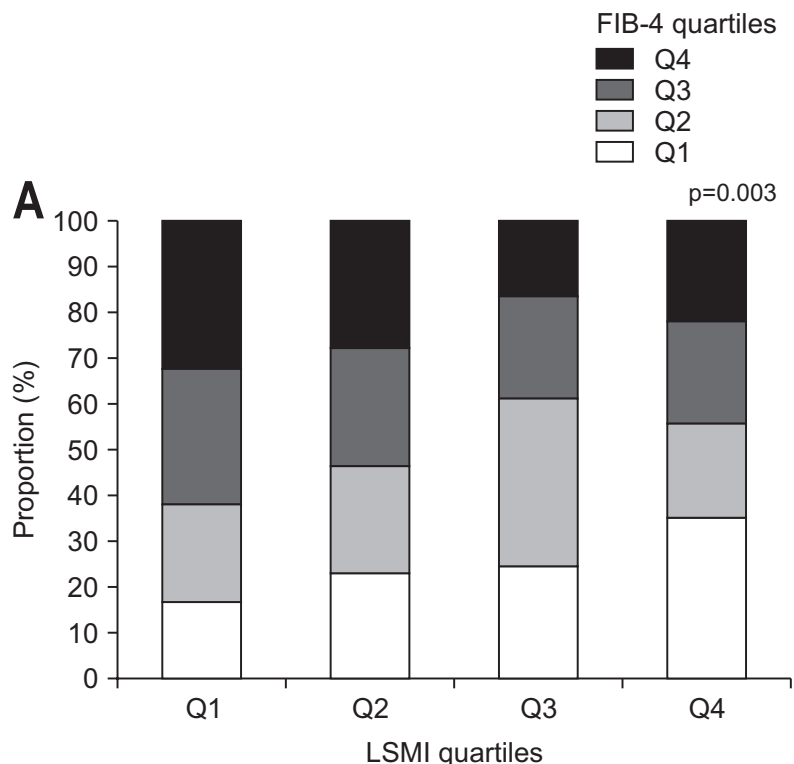

FIB-4 $<1.45$

FIB-4 $\geq 1.45$

B

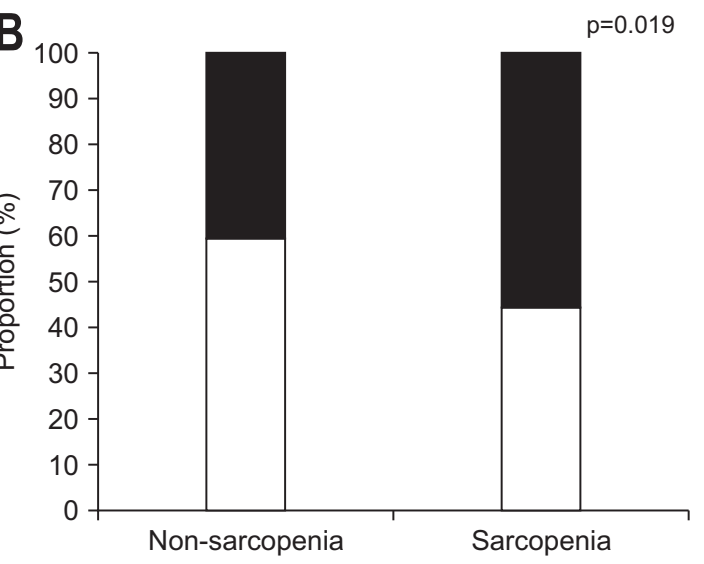

Fig. 3. Association between muscle mass and fibrotic burden by quartile stratification analysis when fibrotic burden was assessed using fibrosis- 4 (FIB-4) index. (A) Muscle mass showed a strong negative association with fibrotic burden $(\mathrm{p}=0.003)$. (B) Sarcopenia patients in the lowest quartile of muscle mass showed a strong association with fibrotic burden, with FIB- $4 \geq 1.45$ ( $\mathrm{p}=0.019$ ). LSMI, lumbar skeletal muscle index. 
and they had a significantly lower L3 skeletal muscle area (mean, $117 \mathrm{~cm}^{2}$ vs $\left.146 \mathrm{~cm}^{2}\right)$, as well as a lower LSMI $\left(41 \mathrm{~cm}^{2} / \mathrm{m}^{2}\right.$ vs 53 $\mathrm{cm}^{2} / \mathrm{m}^{2}$; all $\left.\mathrm{p}<0.05\right)$. Subjects with sarcopenia had more fibrotic liver than those without (mean FIB-4 index, 1.63 vs 1.41; NFS, -0.34 vs -0.66 ; all $\mathrm{p}<0.05$ ). Among the subgroup who had available LS values, as measured by TE $(n=113)$ (Supplementary Table 1), the mean LS value was higher in subjects with sarcopenia than in those without (6.4 $\mathrm{kPa}$ vs $4.9 \mathrm{kPa}$ ), although the difference was not statistically significant $(\mathrm{p}=0.159)$.

\section{Association between muscle mass and fibrotic burden}

Associations between muscle mass and fibrotic burden using FIB-4 index and NFS were analyzed using simple linear regression analysis. Muscle mass assessed using LSMI showed a strong negative association with fibrotic burden assessed using FIB-4 index ( $p=0.023)$, whereas the association between LSMI and NFS was not statistically significant ( $p=0.398$ ) (Fig. 2).

The LSMI by CT scan showed a strong negative association with fibrotic burden by FIB-4 index (Fig. 3). When muscle mass, measured using CT, was stratified into quartiles, 77 (24.9\%), 78 (25.2\%), 76 (24.6\%), and 78 (25.2\%) subjects were stratified into Q1 (lowest quartile, LSMI range: 25.40 to $42.81 \mathrm{~cm}^{2} / \mathrm{m}^{2}$ ), Q2 (lowintermediate quartile, LSMI range: 42.90 to $48.96 \mathrm{~cm}^{2} / \mathrm{m}^{2}$ ), Q3 (high-intermediate quartile, LSMI range: 49.18 to $56.35 \mathrm{~cm}^{2} / \mathrm{m}^{2}$ ), and Q4 (highest quartile, LSMI range: 56.44 to $73.95 \mathrm{~cm}^{2} / \mathrm{m}^{2}$ ), respectively. Similarly, when fibrotic burden by FIB-4 index was stratified by quartile, 77 (24.9\%), 77 (24.9\%), 78 (25.2\%), and 77
(24.9\%) subjects were stratified into Q1 (lowest quartile, FIB-4 range: 0.44 to 0.99), Q2 (low-intermediate quartile, FIB-4 range: 0.99 to 1.30 ), Q3 (high-intermediate quartile, FIB-4 range: 1.30 to 1.87), and Q4 (highest quartile, FIB-4 range: 1.89 to 4.06), respectively. The quartile stratification of fibrotic burden and muscles showed a significantly association $(\mathrm{p}=0.003)$. When an FIB4 index value of 1.45 , the cutoff value for ruling out advanced fibrosis, was used, the proportion of patients with FIB- $4 \geq 1.45$ in the subgroup with sarcopenia was significantly higher than that in the subgroup without sarcopenia (56.0\% vs $40.6 \%$; $p=0.019$ ).

\section{Independent predictors of sarcopenia}

Among the measured variables, age, BMI, ALT level, FIB-4 index, and NFS showed significant correlation with the risk of sarcopenia in univariate analysis (all $\mathrm{p}<0.05$ ) (Table 2). Because age and ALT are constituent variables of the FIB-4 index, they were not incorporated into the multivariate analysis. Similarly, because BMI is a constituent variable of NFS, it was not incorporated into the multivariate analysis. However, because gender is a significant predictor of sarcopenia, we included gender in the multivariate analysis, in spite of statistical insignificance in univariate analysis ( $\mathrm{p}=0.167)$.

When FIB-4 index was used for adjustment, female sex and higher BMI were independently associated with a reduced risk of sarcopenia after adjustment (odds ratio [OR], 0.388; 95\% confidence interval [CI], 0.199 to $0.755 ; \mathrm{p}=0.005$ for female sex and OR, 0.704; 95\% CI, 0.618 to 0.801; $\mathrm{p}<0.001$ for BMI), whereas

Table 2. Independent Predictors of Sarcopenia

\begin{tabular}{|c|c|c|c|c|}
\hline \multirow{2}{*}{ Variable } & \multicolumn{2}{|l|}{ Univariate } & \multicolumn{2}{|c|}{ Multivariate } \\
\hline & OR $(95 \%$ CI $)$ & $\mathrm{p}$-value & OR $(95 \% \mathrm{CI})$ & p-value \\
\hline Age & $1.075(1.045-1.106)$ & $<0.001$ & - & - \\
\hline Female sex & $0.657(0.362-1.192)$ & 0.167 & $0.388(0.199-0.755)$ & 0.005 \\
\hline Body mass index, $\mathrm{kg} / \mathrm{m}^{2}$ & $0.743(0.660-0.838)$ & $<0.001$ & $0.704(0.618-0.801)$ & $<0.001$ \\
\hline Hypertension & $0.927(0.522-1.644)$ & 0.794 & - & - \\
\hline Fasting glucose & $1.000(0.993-1.007)$ & 0.933 & - & - \\
\hline Aspartate aminotransferase, IU/L & $0.987(0.961-1.014)$ & 0.328 & - & - \\
\hline Alanine aminotransferase, IU/L & $0.975(0.953-0.997)$ & 0.026 & - & - \\
\hline Gamma glutamyl-transpeptidase, IU/L & $0.993(0.984-1.001)$ & 0.097 & - & - \\
\hline Serum creatinine, $\mathrm{mg} / \mathrm{dL}$ & $1.284(0.852-1.937)$ & 0.232 & - & - \\
\hline Platelet count, $10^{9} / \mathrm{L}$ & $0.997(0.993-1.002)$ & 0.283 & - & - \\
\hline Prothrombin time, INR & $11.488(0.471-280.000)$ & 0.134 & - & - \\
\hline Total cholesterol, mg/dL & $0.996(0.989-1.002)$ & 0.193 & - & - \\
\hline Triglycerides, mg/dL & $0.997(0.993-1.000)$ & 0.072 & - & - \\
\hline High-density lipoprotein cholesterol, mg/dL & $1.003(0.981-1.026)$ & 0.773 & - & - \\
\hline Low-density lipoprotein cholesterol, mg/dL & $0.997(0.989-1.004)$ & 0.385 & - & - \\
\hline HbA1c, \% & $1.139(0.918-1.412)$ & 0.237 & - & - \\
\hline Fibrosis-4 index & $2.195(1.426-3.379)$ & 0.003 & $1.817(1.180-2.797)$ & 0.007 \\
\hline
\end{tabular}

OR, odds ratio; CI, confidence interval; INR, international normalized ratio; HbA1c, glycosylated hemoglobin. 
higher FIB-4 index was independently associated with an increased risk of sarcopenia (OR, 1.817; 95\% CI, 1.180 to 2.797; $\mathrm{p}=0.007)$. When age and ALT, the respective variables of FIB4 index, were adjusted, older age was independently associated with the increased risk of sarcopenia (OR, 1.086; 95\% CI, 1.051 to $1.122 ; \mathrm{p}<0.001)$, whereas female sex and higher BMI were independently associated with a reduced risk of sarcopenia (OR, 0.280 ; $95 \%$ CI, 0.138 to $0.568 ; \mathrm{p}<0.001$ for female sex and OR, 0.710 ; 95\% CI, 0.620 to 0.814 ; $\mathrm{p}<0.001$ for BMI) (Supplementary Table 2). When NFS was used for adjustment, only higher NFS was independently associated with an increased risk of sarcopenia (OR, 1.322; 95\% CI, 1.028 to 1.698; $\mathrm{p}=0.029$ ) (Table 2).

In the subgroup with LS values available, female sex (OR, 0.102; 95\% CI, 0.012 to 0.897; $\mathrm{p}=0.040$ ) and higher BMI (OR, $0.678 ; 95 \% \mathrm{CI}, 0.522$ to $0.894 ; \mathrm{p}=0.006$ ) were independently associated with a reduced risk of sarcopenia after adjustment, whereas LS tended to be associated with sarcopenia, with borderline statistical significance $(\mathrm{p}=0.086)$ (Supplementary Table 3).

\section{Degree of liver fibrosis and sarcopenic status stratified by BMI and gender}

To investigate the association between the degree of liver fibrosis and sarcopenic status without the confounding influences of obesity and gender, we stratified the study population using a BMI cutoff value of $25 \mathrm{~kg} / \mathrm{m}^{2}$ ( $\mathrm{n}=165$ [53.4\%] with a BMI $<25 \mathrm{~kg} / \mathrm{m}^{2}$ and $\mathrm{n}=144$ [46.6\%] with a BMI $\geq 25 \mathrm{~kg} / \mathrm{m}^{2}$ ), as well as gender (men, $n=215$ [69.6\%]; women, $n=94$ [30.4\%]) (Fig. 4).

Among the men, the FIB-4 index of patients with sarcopenia $(n=57,26.5 \%)$ tended to be higher than that of those without $(n=158,73.5 \%)$, although statistical significance was not
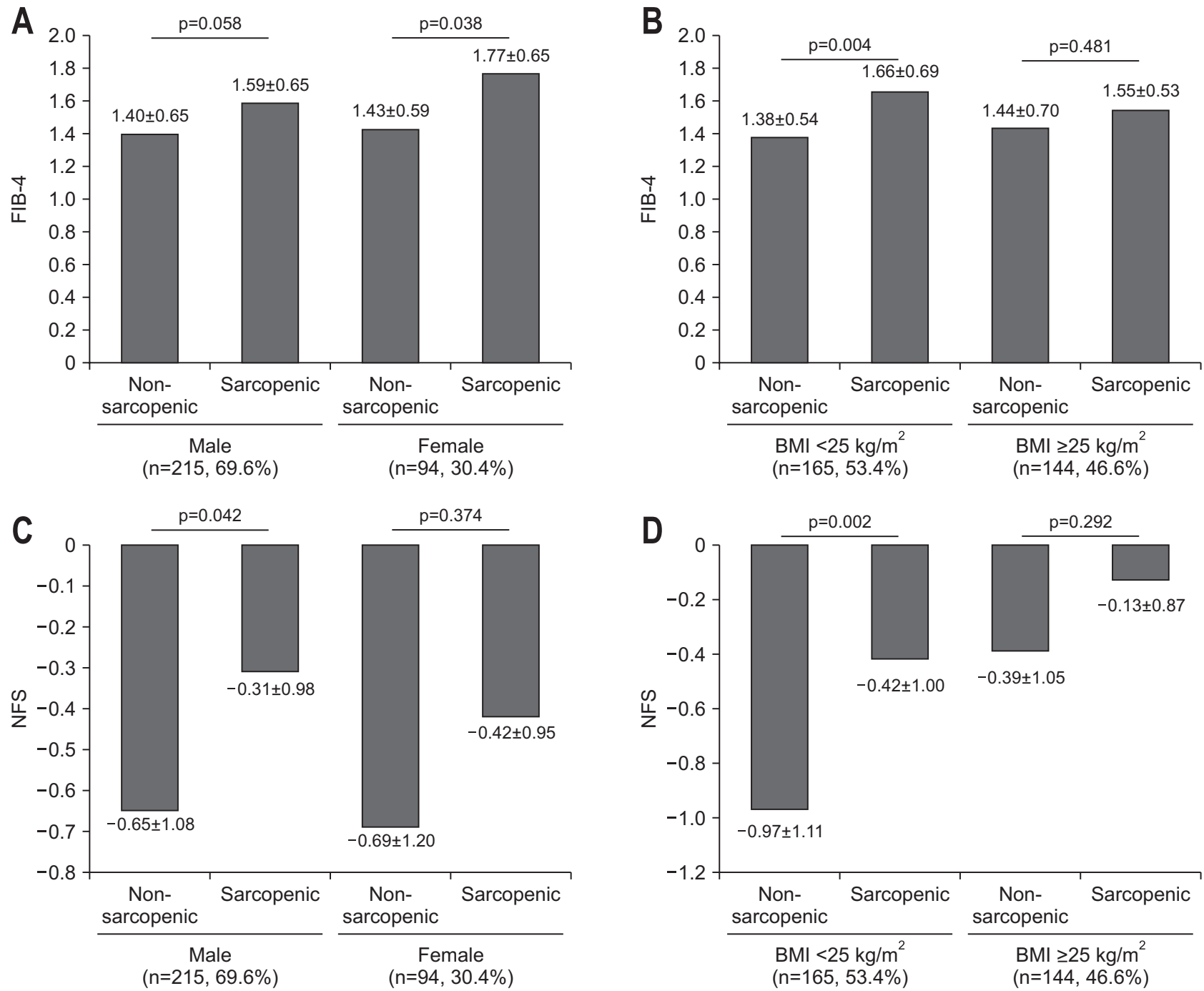

Fig. 4. Fibrotic burden was assessed using the fibrosis-4 (FIB-4) index and NAFLD fibrosis score (NFS) according to sarcopenic status after stratification according to sex and BMI $\left(<25\right.$ and $\left.\geq 25 \mathrm{~kg} / \mathrm{m}^{2}\right)$. When analyzed using FIB-4, sarcopenic subjects had a significantly higher fibrotic burden than nonsarcopenic subjects in the subgroups with female sex (A) and BMI $<25 \mathrm{~kg} / \mathrm{m}^{2}$ (B) (all p<0.05). When analyzed using NFS, sarcopenic subjects had a significantly higher fibrotic burden than nonsarcopenic subjects in subgroups of male sex (C) and a BMI $<25 \mathrm{~kg} / \mathrm{m}^{2}$ (D) (all p<0.05). NAFLD, nonalcoholic fatty liver disease; BMI, body mass index. 
reached (mean, 1.59 vs 1.40; $\mathrm{p}=0.058$ ) (Fig. 4A). However, when analyzed using NFS, among the men, the NFS of men with sarcopenia $(n=57,26.5 \%)$ was significantly higher than that of those without ( $\mathrm{n}=158,73.5 \%$ ) (mean, -0.31 vs $-0.65 ; \mathrm{p}=0.042$ ) (Fig. 4C). In addition, among the women, the FIB-4 index of patients with sarcopenia $(n=18,19.1 \%)$ was significantly higher than that of those without ( $\mathrm{n}=76,80.9 \%$ ) (mean, 1.77 vs 1.43 ; $\mathrm{p}=0.038$ ) (Fig. 4A). However, when analyzed using NFS, among the women, the NFS was statistically similar between women with sarcopenia $(n=18,19.1 \%)$ and those without sarcopenia ( $n=76,80.9 \%$ ) (mean, -0.42 vs $-0.69 ; \mathrm{p}=0.374$ ) (Fig. 4C).

Among the 165 subjects with a BMI of $<25 \mathrm{~kg} / \mathrm{m}^{2}$, patients with sarcopenia ( $n=54,32.7 \%$ ) had significantly higher FIB-4 index and NFS values than those without $(n=111,67.3 \%)$ (mean FIB-4, 1.66 vs $1.38 ; p=0.004$ and NFS, -0.42 vs $-0.97 ; p=0.002$, respectively) (Fig. 4B and D). Among the 144 patients with a BMI $\geq 25 \mathrm{~kg} / \mathrm{m}^{2}$, FIB-4 index and NFS were statistically similar between patients with sarcopenia $(n=123,85.4 \%)$ and those without ( $\mathrm{n}=21,14.6 \%$ ) (mean FIB-4, 1.55 vs 1.44 and NFS, -0.13 vs -0.39 , respectively; all $\mathrm{p}<0.05$ ) (Fig. $4 \mathrm{~B}$ and $\mathrm{D}$ ).

When the subgroup with the LS value available was assessed, LS values were statistically similar between patients with sarcopenia and those without (all p>0.05) (Supplementary Fig. 1).

\section{DISCUSSION}

In the present study, we found that around one quarter of diabetic patients had sarcopenia (24.3\%), and that muscle mass, as measured by LSMI, was significantly negatively associated with fibrotic burden in liver, as measured by FIB-4 index ( $\mathrm{p}=0.003)$. In addition, we found that female sex (OR, 0.388) and higher BMI (OR, 0.704) were independently associated with a reduced risk of sarcopenia, whereas higher fibrotic burden in liver, as reflected by higher FIB-4 index (OR, 1.817), was independently associated with an increased risk of sarcopenia in patients with diabetes. However, when NFS was used for adjustment, only higher NFS was independently associated with an increased risk of sarcopenia after adjustment (OR, 1.322). When the study population was stratified according to gender, patients with sarcopenia tended to have higher FIB-4 index and NFS values than those without in the male subgroup (mean FIB-4, 1.59 vs 1.40 and NFS, -0.31 vs -0.65 ), whereas FIB-4 index was significantly higher in patients with sarcopenia in the female subgroup (mean, 1.77 vs $1.43 ; \mathrm{p}=0.038)$. When the study population was stratified according to BMI, FIB-4 index and NFS were significantly higher in patients with sarcopenia than in those without in the subgroup with a BMI $<25 \mathrm{~kg} / \mathrm{m}^{2}$ (mean FIB-4, 1.66 vs 1.38 and NFS, -0.42 vs -0.97). However, the FIB-4 index and NFS were statistically similar between patients with and without sarcopenia in the subgroup with a BMI $\geq 25 \mathrm{~kg} / \mathrm{m}^{2}$.

Our study had several strengths. Firstly, we measured skeletal muscle mass using CT. Several previous studies have shown that
CT scanning is more accurate than DXA or BIA in the quantitative measurement of skeletal muscle mass. ${ }^{3,23}$ In addition, CTmeasured values of skeletal muscle mass have been associated with qualitative measures of skeletal muscle function, such as strength and physical performance; ${ }^{3}$ the main pitfalls of DXA and BIA in this regard are their dependency on assumptions regarding the density and hydration fraction of fat-free mass, which can be biased in patients with advanced liver disease, because they tend to retain water. ${ }^{3,23}$ Furthermore, to assess skeletal muscle mass using DXA or BIA, specific equipment is required, whereas CT scanning is readily available in most institutions. ${ }^{24}$ Thus, the results of our study might be readily applicable in clinical practice, helping physicians to identify patients with sarcopenia who are at risk of liver fibrosis progression and provide appropriate medical interventions, such as exercise and protein supplements.

Secondly, our study focused on diabetic patients. In chronic hepatitis B and hepatitis C virus infections, as well as in NAFLD, T2DM has been related to liver fibrosis, ${ }^{25,26}$ and the prevalence of sarcopenia is higher in diabetic patients. ${ }^{27}$ Several recent studies have confirmed an independent association between fibrotic burden in the liver and sarcopenia in NAFLD and hepatitis B virus cohorts. ${ }^{11-14}$ In these studies, the independent association between liver fibrosis and sarcopenia was found to be independent of insulin resistance, although few studies have focused on patients with diabetes. In the current study, which had quite a large sample size $(n=309)$, homogeneous diabetic patients were recruited to exclude the influence of T2DM itself on liver fibrosis. This may have ensured statistical reliability in this specific population. In this regard, a previous study reported the association between liver fibrosis and sarcopenia, as assessed using body circumference, in a diabetic cohort. The results showed that higher waist-to-calf circumference ratio, which is related to sarcopenic obesity, was associated with an increased risk of NAFLD and liver fibrosis, independent of insulin resistance. ${ }^{28}$

Thirdly, the results of our current study were supported by several recent studies, ${ }^{10-14,28,29}$ which indicated that liver fibrosis progression is significantly associated with skeletal muscle loss, regardless of various concomitant medical illnesses. Indeed, there is likely a significant association between liver fibrosis and sarcopenia in patients with NAFLD, although this has not yet been confirmed. ${ }^{11}$ Thus, considering that both fatty liver and T2DM involve insulin resistance, ${ }^{30}$ it is not surprising that fibrosis is significantly associated with sarcopenia in diabetic patients as well. The mechanism of the independent association between the degree of sarcopenia and fibrotic burden in the liver was not revealed in the present cross-sectional study. However, the underlying mechanisms of sarcopenia in the NAFLD and T2DM cohorts may be related to insulin resistance, vitamin D deficiency, myostatin, sedentary lifestyle, etc. ${ }^{31}$ Thus, our study again indicated the need for future studies to investigate whether exercise, nutritional supplementation, and medica 
treatment of sarcopenia can ameliorate liver fibrosis. ${ }^{29,32,33}$

Fourthly, previous studies have indicated that the prevalence of sarcopenia is higher in women than in men, ${ }^{34}$ perhaps because there is a higher prevalence of NAFLD in women. ${ }^{11}$ However, in the present study, we found that the association between liver fibrosis and sarcopenia was consistent regardless of gender, although it did show borderline statistical significance men ( $\mathrm{p}=0.058$ ). This might indicate that the clinical implications of sarcopenia and corresponding liver fibrosis differ between genders, and that the difference in liver fibrotic burden between patients with sarcopenia and those without is only significant in the subgroups with a BMI $<25 \mathrm{~kg} / \mathrm{m}^{2}$. This might indicate that subjects with sarcopenic obesity have a similar risk of liver fibrosis progression than those with non-sarcopenic obesity. However, because subjects with sarcopenic obesity have poorer prognosis than those with non-sarcopenic obesity, including poorer physical performance and higher risk of cardiovascular disease and metabolic syndrome, ${ }^{6,7,35,36}$ future studies should assess whether long-term liver-related outcomes are similar between these groups. In addition, further studies might be required to define a new BMI cutoff value that can reveal the influence of sarcopenia among subjects with obesity.

There were also several unresolved issues in the present study. Firstly, because this was a cross-sectional study, we could not investigate the influence of insulin resistance, anti-diabetic medications, or illness duration. In this regard, several previous studies have shown that some anti-diabetic drugs can induce muscle atrophy, ${ }^{37,38}$ so further studies are required to resolve this issue. Secondly, due to the cross-sectional and retrospective nature of our study, we could not evaluate the influence of sarcopenia on the prognosis of patients with of T2DM or assess the longitudinal dynamic association between liver fibrosis progression and changes in muscle mass during the natural course or after therapeutic interventions, such as lifestyle modification, exercise, and weight loss. However, based on our results, we might cautiously recommend exercise or protein supplements when muscle mass falls below cutoff levels. Thirdly, we used CT to measure skeletal muscle mass. Although muscle mass assessed using CT can also reflect muscle function such as muscle strength and physical performance, ${ }^{3}$ further validation studies are required. Fourthly, due to differences in scanning thickness depending on the number of channels in varying CT scans, areas at the mid L3 level might vary slightly for each machine. Fifthly, our study is not free from potential selection bias, because only subjects who could afford to pay the cost for the comprehensive medical health check-up including CT scans were recruited. Fifthly, some results were discordant according to the use of FIB-4 index or NFS, probably due to the different constituent variables and overall small sample size. Further validation study is required to resolve this issue. Lastly, in the subgroup analysis of patients with available TE results, the association between fibrotic burden, expressed as LS and sarcopenia, was not statistically significant, probably due to the insufficient sample size. Because TE can accurately assess fibrotic burden in the liver, further studies using TE are warranted.

In conclusion, the present retrospective study found that sarcopenia is independently associated with fibrotic burden in patients with T2DM. Further well-designed, prospective, longitudinal studies are warranted to elucidate the complex relationship between sarcopenia and liver fibrosis progression.

\section{CONFLICTS OF INTEREST}

No potential conflict of interest relevant to this article was reported.

\section{ACKNOWLEDGEMENTS}

This work was supported by a grant from the Korea Health Technology R\&D Project through the Korea Health Industry Development Institute, which is funded by the Ministry of Health Welfare, Republic of Korea (HI15C2859) (J.Y.P) and the Basic Science Research Program through the National Research Foundation of Korea (NRF) funded by the Ministry of Science, ICT \& Future Planning (2019R1A2C4070136) (S.U.K).

\section{AUTHOR CONTRIBUTIONS}

Conceptualization: M.J.S., J.Y.P., S.U.K. Methodology: T.S.L., M.Y.J., H.W.L., B.K.K., D.Y.K., S.H.A., K.H.H., J.Y.P. Formal analysis: M.J.S. Funding acquisition: J.Y.P. Project administration: J.Y.P., S.U.K. Visualization: M.J.S. Writing - original draft: M.J.S., J.Y.P., S.U.K. Writing - review and editing: M.J.S., J.Y.P., S.U.K. Approval of final manuscript: all authors.

\section{ORCID}

Min Je Sung

Tae Seop Lim

Mi Young Jeon

Hye Won Lee

Beom Kyung Kim

Do Young Kim

Sang Hoon Ahn

Kwang-Hyub Han

Jun Yong Park

Seung Up Kim https://orcid.org/0000-0001-5395-8851

https://orcid.org/0000-0002-4578-8685

https://orcid.org/0000-0002-3980-4503

https://orcid.org/0000-0002-3552-3560

https://orcid.org/0000-0002-5363-2496

https://orcid.org/0000-0002-8327-3439

https://orcid.org/0000-0002-3629-4624

https://orcid.org/0000-0003-3960-6539

https://orcid.org/0000-0001-6324-2224

https://orcid.org/0000-0002-9658-8050

\section{REFERENCES}

1. Cruz-Jentoft AJ, Baeyens JP, Bauer JM, et al. Sarcopenia: European consensus on definition and diagnosis: report of the European Working Group on Sarcopenia in Older People. Age Ageing 2010;39:412-423. 
2. Chen LK, Liu LK, Woo J, et al. Sarcopenia in Asia: consensus report of the Asian Working Group for Sarcopenia. J Am Med Dir Assoc 2014;15:95-101.

3. Itoh S, Shirabe K, Yoshizumi T, et al. Skeletal muscle mass assessed by computed tomography correlates to muscle strength and physical performance at a liver-related hospital experience. Hepatol Res 2016;46:292-297.

4. The Lancet Diabetes Endocrinology. Sarcopenia: a fate worth challenging. Lancet Diabetes Endocrinol 2014;2:183.

5. Kim TN, Park MS, Yang SJ, et al. Prevalence and determinant factors of sarcopenia in patients with type 2 diabetes: the Korean Sarcopenic Obesity Study (KSOS). Diabetes Care 2010;33:14971499.

6. Stephen WC, Janssen I. Sarcopenic-obesity and cardiovascular disease risk in the elderly. J Nutr Health Aging 2009;13:460-466.

7. Lim S, Kim JH, Yoon JW, et al. Sarcopenic obesity: prevalence and association with metabolic syndrome in the Korean Longitudinal Study on Health and Aging (KLoSHA). Diabetes Care 2010;33:1652-1654.

8. Cruz-Jentoft AJ, Landi F, Topinková E, Michel JP. Understanding sarcopenia as a geriatric syndrome. Curr Opin Clin Nutr Metab Care 2010;13:1-7.

9. Rinella ME. Nonalcoholic fatty liver disease: a systematic review. JAMA 2015;313:2263-2273.

10. Hong HC, Hwang SY, Choi HY, et al. Relationship between sarcopenia and nonalcoholic fatty liver disease: the Korean Sarcopenic Obesity Study. Hepatology 2014;59:1772-1778.

11. Lee YH, Kim SU, Song K, et al. Sarcopenia is associated with significant liver fibrosis independently of obesity and insulin resistance in nonalcoholic fatty liver disease: nationwide surveys (KNHANES 2008-2011). Hepatology 2016;63:776-786.

12. Koo BK, Kim D, Joo SK, et al. Sarcopenia is an independent risk factor for non-alcoholic steatohepatitis and significant fibrosis. $\mathrm{J}$ Hepatol 2017;66:123-131.

13. Lee YH, Jung KS, Kim SU, et al. Sarcopaenia is associated with NAFLD independently of obesity and insulin resistance: nationwide surveys (KNHANES 2008-2011). J Hepatol 2015;63:486-493.

14. Han E, Lee YH, Kim BK, et al. Sarcopenia is associated with the risk of significant liver fibrosis in metabolically unhealthy subjects with chronic hepatitis B. Aliment Pharmacol Ther 2018;48:300312.

15. American Diabetes Association. Standards of medical care in diabetes: 2014. Diabetes Care 2014;37 Suppl 1:S14-S80.

16. Baracos VE, Reiman T, Mourtzakis M, Gioulbasanis I, Antoun S. Body composition in patients with non-small cell lung cancer: a contemporary view of cancer cachexia with the use of computed tomography image analysis. Am J Clin Nutr 2010;91:1133S1137 S.

17. Prado CM, Lieffers JR, McCargar LJ, et al. Prevalence and clinical implications of sarcopenic obesity in patients with solid tumours of the respiratory and gastrointestinal tracts: a population-based study. Lancet Oncol 2008;9:629-635.
18. American Diabetes Association. Diagnosis and classification of diabetes mellitus. Diabetes Care 2014;37 Suppl 1:S81-S90.

19. Vallet-Pichard A, Mallet V, Nalpas B, et al. FIB-4: an inexpensive and accurate marker of fibrosis in HCV infection: comparison with liver biopsy and fibrotest. Hepatology 2007;46:32-36.

20. Angulo P, Hui JM, Marchesini G, et al. The NAFLD fibrosis score: a noninvasive system that identifies liver fibrosis in patients with NAFLD. Hepatology 2007;45:846-854.

21. Kim SU, Ahn SH, Park JY, et al. Liver stiffness measurement in combination with noninvasive markers for the improved diagnosis of B-viral liver cirrhosis. J Clin Gastroenterol 2009;43:267-271.

22. Chon YE, Jung KS, Kim SU, et al. Controlled attenuation parameter (CAP) for detection of hepatic steatosis in patients with chronic liver diseases: a prospective study of a native Korean population. Liver Int 2014;34:102-109.

23. Giusto M, Lattanzi B, Albanese C, et al. Sarcopenia in liver cirrhosis: the role of computed tomography scan for the assessment of muscle mass compared with dual-energy X-ray absorptiometry and anthropometry. Eur J Gastroenterol Hepatol 2015;27:328-334.

24. Hiraoka A, Aibiki T, Okudaira T, et al. Muscle atrophy as presarcopenia in Japanese patients with chronic liver disease: computed tomography is useful for evaluation. J Gastroenterol 2015;50:1206-1213.

25. Papatheodoridis GV, Chrysanthos N, Savvas S, et al. Diabetes mellitus in chronic hepatitis B and C: prevalence and potential association with the extent of liver fibrosis. J Viral Hepat 2006;13:303310.

26. Angulo P, Keach JC, Batts KP, Lindor KD. Independent predictors of liver fibrosis in patients with nonalcoholic steatohepatitis. Hepatology 1999;30:1356-1362.

27. Liccini A, Malmstrom TK. Frailty and sarcopenia as predictors of adverse health outcomes in persons with diabetes mellitus. J Am Med Dir Assoc 2016;17:846-851.

28. Choe EY, Lee YH, Choi YJ, et al. Waist-to-calf circumstance ratio is an independent predictor of hepatic steatosis and fibrosis in patients with type 2 diabetes. J Gastroenterol Hepatol 2018;33:10821091.

29. Hanai T, Shiraki M, Nishimura K, et al. Sarcopenia impairs prognosis of patients with liver cirrhosis. Nutrition 2015;31:193-199.

30. Bugianesi E, Gastaldelli A, Vanni E, et al. Insulin resistance in non-diabetic patients with non-alcoholic fatty liver disease: sites and mechanisms. Diabetologia 2005;48:634-642.

31. Bhanji RA, Narayanan P, Allen AM, Malhi H, Watt KD. Sarcopenia in hiding: the risk and consequence of underestimating muscle dysfunction in nonalcoholic steatohepatitis. Hepatology 2017;66:2055-2065.

32. Kawaguchi T, Shiba N, Takano Y, Maeda T, Sata M. Hybrid training of voluntary and electrical muscle contractions decreased fasting blood glucose and serum interleukin-6 levels in elderly people: a pilot study. Appl Physiol Nutr Metab 2011;36:276-283.

33. Kawaguchi T, Shiba N, Maeda T, et al. Hybrid training of voluntary and electrical muscle contractions reduces steatosis, insulin 
resistance, and IL-6 levels in patients with NAFLD: a pilot study. J Gastroenterol 2011;46:746-757.

34. Janssen I, Heymsfield SB, Ross R. Low relative skeletal muscle mass (sarcopenia) in older persons is associated with functional impairment and physical disability. J Am Geriatr Soc 2002;50:889-896.

35. Chung JY, Kang HT, Lee DC, Lee HR, Lee YJ. Body composition and its association with cardiometabolic risk factors in the elderly: a focus on sarcopenic obesity. Arch Gerontol Geriatr 2013;56:270-278.

36. Rolland Y, Lauwers-Cances V, Cristini C, et al. Difficulties with physical function associated with obesity, sarcopenia, and sarcopenic-obesity in community-dwelling elderly women: the EPIDOS (EPIDemiologie de l'OSteoporose) Study. Am J Clin Nutr 2009;89:1895-1900.

37. Cetrone M, Mele A, Tricarico D. Effects of the antidiabetic drugs on the age-related atrophy and sarcopenia associated with diabetes type II. Curr Diabetes Rev 2014;10:231-237.

38. Campins L, Camps M, Riera A, Pleguezuelos E, Yebenes JC, SerraPrat M. Oral drugs related with muscle wasting and sarcopenia: a review. Pharmacology 2017;99:1-8. 